\title{
Association of age and colostrum discarding with exclusive breastfeeding in Ethiopia: systematic review and meta-analyses
}

Sisay Mulugeta Alemu ${ }^{1}$, Yihun Mulugeta Alemu ${ }^{2}$, Tesfa Dejenie Habtewold ${ }^{3}$

${ }^{1}$ Mental Health and Psychosocial Support Program, International Medical Corps, Dolo Ado, Ethiopia

${ }^{2}$ Department of Public Health, Bahir Dar University, Bahir Dar, Ethiopia

${ }^{3}$ Department of Epidemiology and Psychiai;try, University of Groningen, Groningen, the Netherlands

Correspondence should be addressed to Sisay Mulugeta Alemu; sisaym8@gmail.com 


\begin{abstract}
Introduction: Even though optimal breastfeeding is important, significantly low percentage of mothers' initiate breastfeeding timely and maintain exclusive breastfeeding for 6 months. The aim of this meta-analyses and systematic review was to investigate whether maternal/caregivers' age, infant age (0-6 months) and discarding colostrum affects timely initiation of breastfeeding (TIBF) and exclusive breastfeeding (EBF) in Ethiopia.
\end{abstract}

Methods: A systematic search of PubMed, SCOPUS, EMBASE, CINHAL, Web of Science and WHO Global Health Library electronic databases was done for all English published articles from 2000 to January 2018, supplemented by manual search of identified articles and grey literatures bibliographies. Two reviewers independently screened, extracted and graded the quality studies using Newcastle-Ottawa Scale (NOS). Heterogeneity was assessed using the $\mathrm{I}^{2}$ and Cochran Chi-square statistics. A weighted inverse variance random-effects model metaanalysis was done.

Result: A total of 37 articles (i.e., 14 studies on TIBF and 23 on EBF) were included. TIBF was associated with colostrum discarding (Odds ratio $(\mathrm{OR})=0.38,95 \% \mathrm{CI}=0.21-0.68)$ but not with maternal/caregivers' age $(\mathrm{OR}=0.98,95 \% \mathrm{CI}=0.83-1.15)$. In addition, colostrum discarding $(\mathrm{OR}=0.56,95 \% \mathrm{CI}=0.37-0.84)$ and infant age $(\mathrm{OR}=1.86,95 \% \mathrm{CI}=1.45-2.39)$ were significantly associated with $\mathrm{EBF}$ but not maternal/caregivers' age $(\mathrm{OR}=1.07,95 \% \mathrm{CI}=0.81$ 1.40).

Conclusion: This meta-analyses indicated absence of association between maternal/caregivers' age and breastfeeding practice. Colostrum discarding was associated with both EBF and TIBE. This evidence could be helpful to counsel all reproductive age mothers and who discard colostrum.

Key words: Breast Feeding, maternal age, infant, review, meta-analysis, Ethiopia 


\section{Introduction}

World Health Organization (WHO) and United Nation Children's Fund (UNICEF) defines timely initiation of breastfeeding (TIBF) as putting a new born to breast within one hour of birth and exclusive breastfeeding (EBF) as feeding infants only human milk through breastfeeding or expressed breast milk and no other liquids or solids, except for drops or syrups with nutritional supplements or medicine. (1) All infants should receive human within the first hour of birth, exclusively breastfed for the first six months and thereafter, nutritionally adequate and safe complementary foods to be introduced with continued breastfeeding for at least two years. ${ }^{(2,3)}$ Breastfeeding is one of the smartest investment that prevents maternal and newborn morbidity and mortality. ${ }^{(4-7)}$ For example, TIBF and EBF prevents $22 \%$ and $60 \%$ of neonatal deaths respectively. ${ }^{(2,8,9)}$ Furthermore, exclusive breastfeeding for a longer duration benefits child neurodevelopment and increase IQ. ${ }^{(10)}$

Despite the aforementioned advantages, significantly low percentage of mothers initiate breastfeeding within the first hour of birth and maintain exclusive breastfeeding for 6 months. Globally, $44 \%$ and $40 \%$ of newborns breastfed with in the first hour and exclusively breastfeed for six months respectively. ${ }^{(4,11)}$ In developing countries, the prevalence of TIBF ranges 22.4 to $52.8 \%{ }^{(17-23)}$ and EBF ranges 10 to $49.1 \% .^{(11,17,18,27,28)}$ In Ethiopia, based on our previous metaanalyses, (unpublished results) the national prevalence of TIBF and EBF is $67.5 \%$ and $60.5 \%$ respectively.

Previous studies have identified several associated factors, including maternal/caregiver's age, newborn age and colostrum discarding, of timely TIBF and EBF. ${ }^{(12-22)}$ Previous studies shows that infant age and colostrum discarding have been associated with late initiation of breastfeeding and nonexclusive breastfeeding. ${ }^{(18,20,23-25)}$ Regarding maternal/caregiver's age, most of the reviewed literatures reveals that older mothers practice $\operatorname{TIBF}^{(14,19,20,26)}$ and $\operatorname{EBF}^{(12,}$ $17,22,27,28)$ higher than young mothers although the age cut-off value varies between studies. Another study, ${ }^{(13)}$ which measured age as a continuous variable, also concludes that increased maternal age positively associated TIBF and EBF. On the contrary, some studies showed that increased maternal age was associated with delayed initiation of breastfeeding and nonexclusive breastfeeding. ${ }^{(15,16)}$ Furthermore, other studies showed absence of association. ${ }^{(21,29)}$ Taken together, inconsistencies persisted and the association is inconclusive. 
Hence, there is an urgent need to synthesize individual studies data to make a better conclusion on the association between maternal age, infant age and colostrum discarding and breastfeeding practice (i.e., TIBF and EBF). So far, several systematic reviews and meta-analyses have been conducted on TIBF and EBF. ${ }^{(14,18,20,30-32)}$ In Ethiopia, only one meta-analysis investigated the association of place of residence and delivery with TIBF. ${ }^{(32)}$ In our previous meta-analyses, (unpublished results) we studied the association between maternal employment, breastfeeding counseling, model of delivery, place of delivery, sex of newborn, antenatal care and postnatal care and breastfeeding practice. We also investigated the association between TIBF and EBF. The present meta-analyses and systematic review aimed to determine whether maternal/caregivers age, infant age and colostrum discharging affects TIBF and EBF in Ethiopia. We hypothesized (i) increased maternal age positively associated with breastfeeding practice due to accumulated experience, (ii) increased infant age negatively associated with exclusive breastfeeding and (iii) colostrum discarding negatively associated with breastfeeding practice.

Following international recommendations, the Ethiopian government has taken steps to improving infant and young child feeding practices. Several national nutritional strategies, ${ }^{(33)}$ guidelines $^{(34)}$ and nutrition programs ${ }^{(35,36)}$ have been developed by Ministry of Health of Ethiopia since 2004. Likewise, the Health Sector Transformation Plan of Ethiopia ${ }^{(37)}$ has targeted to increase exclusive breast feeding to $72 \%$ by 2020 . Furthermore, Ethiopia has recently started celebrating world breastfeeding week every year. ${ }^{(38)}$ However, TIBF and EBF coverage is still below the WHO recommendation and attributed to several factors. This meta-analyses information could be valuable to provide updated evidence to develop national guidelines and strategies. 


\section{Methods}

\section{Protocol registration and publication}

The protocol has been registered with the University of York Centre for Reviews and Dissemination International prospective register of systematic reviews (PROSPERO) (http://www.crd.york.ac.uk/PROSPERO/display_record.asp?ID=CRD42017056768) and published. ${ }^{(39)}$

\section{Data source and search strategy}

For all available publications, systematic search of PubMed, SCOPUS, EMBASE, Cumulative Index to Nursing and Allied Health Literature (CINAHL), Web of Science and WHO Global Health Library electronic databases was done. In addition, bibliographies of identified articles and grey literatures were hand-searched. A comprehensive search strategy was developed for each database in consultation with a medical information specialist (Supplementary file 1).

\section{Eligibility criteria}

All studies published in English from 2000 to January 2018 were included. In addition, observational studies (cross-sectional, case-control, cohort, survey and surveillance reports) conducted in Ethiopia were included. However, studies on preterm newborn infants, infants in neonatal intensive care unit or a special care baby unit, low birth weight and mothers or infants with medical problems were excluded. Further, commentaries, anonymous reports, letters, duplicate studies, editorials, qualitative studies and citations without full text were excluded.

\section{Study screening and selection}

All studies obtained from databases and manual search were exported to EndNote citation manager. The title and abstract of all studies were screened by reviewers (SM \& TD) independently. Agreement between the reviewers, as measured by Cohen's Kappa, was 0.76. Any disagreement was resolved by discussion. When consensus could not be reached, a third reviewer approved the final list of retained studies. A full-text review was performed by two independent investigators (SM \& TD). 


\section{Quality assessment and data extraction}

Newcastle-Ottawa Scale (NOS), which has good inter-rater reliability and validity, was used to assess the quality of studies and for potential publication bias. ${ }^{(40,41)}$ In addition, to define outcome measurements, WHO infant and young child feeding practice guideline was strictly followed. Based on previous systematic review report, ${ }^{(14,42,43)}$ maternal/Caregiver's age was dichotomized as $\geq 25$ versus $<25$ years old whereas infant age was dichotomized as $\leq 3$ versus 3 to 6 months age. Joanna Briggs Institute (JBI) tool was used to extract the following data: study area (region and place), method (design), population, number of mothers (calculated sample size and participated in actual study) and cross-tabulated data. Geographic regions were categorized based on the current Federal Democratic Republic of Ethiopia administrative structure (Supplementary file 2). Discrepancies were resolved by consensus and cross-checking with the full-text.

\section{Statistical analysis}

A weighted inverse variance random-effects model meta-analyses was implemented. Publication bias was assessed by visual inspection of funnel plot and Egger's regression test for funnel plot asymmetry using standard error as a predictor in mixed-effects meta-regression model at p-value threshold $\leq 0.01$. $^{(44)}$ Duval and Tweedie trim-and-fill method ${ }^{(45)}$ was used if we found asymmetric funnel which indicate publication bias. Heterogeneity was assessed by Cochran's Q $\mathrm{X}^{2}$ test $(\mathrm{p}$-value $\leq 0.05)$ and $\mathrm{I}^{2}$ statistics (reference value $\left.>80 \%\right) .{ }^{(39)}$ The data was analyzed using "metaphor" packages in R software version 3.2.1 for Window.

\section{Data synthesis and reporting}

We analyzed the data in two groups of outcome measurements: TIBF and EBF. Results for each variable were shown using forest plots. Preferred Reporting Items for Systematic Reviews and Meta-Analyses (PRISMA) guideline was strictly followed (Supplementary file 3).

\section{Minor post hoc protocol changes}

Before analysis was done, we made the following changes to our methods from the published protocol. We added the Joanna Briggs Institute (JBI) tool to extract the data. In addition, we used Duval and Tweedie trim-and-fill method to manage publication bias. 


\section{Result}

\section{Search results}

We obtained 169 articles from PubMed, 24 from EMBASE, 200 from Web of Science, 85 from SCOPUS and 5 from other (CINHAL and WHO Global Health Library) electronic database searching. Forty-eight additional articles were found through a manual search of reference lists of included articles. After removing duplicates and screening of titles and abstracts, full-text of 82 studies were reviewed to assess eligibility. Forty-five articles were excluded after a full-text review due to several reasons: 19 studies on complementary feeding, 3 on pre-lacteal feeding, 3 on malnutrition, 19 with different variables of interest and one project review report. As a result, 37 articles (i.e., 14 studies on timely initiation of breastfeeding and 23 on exclusive breastfeeding) fulfilled the inclusion criteria and were included in the meta-analyses. The PRISMA flow diagram of literature screening and selection process is shown in figure 1. 


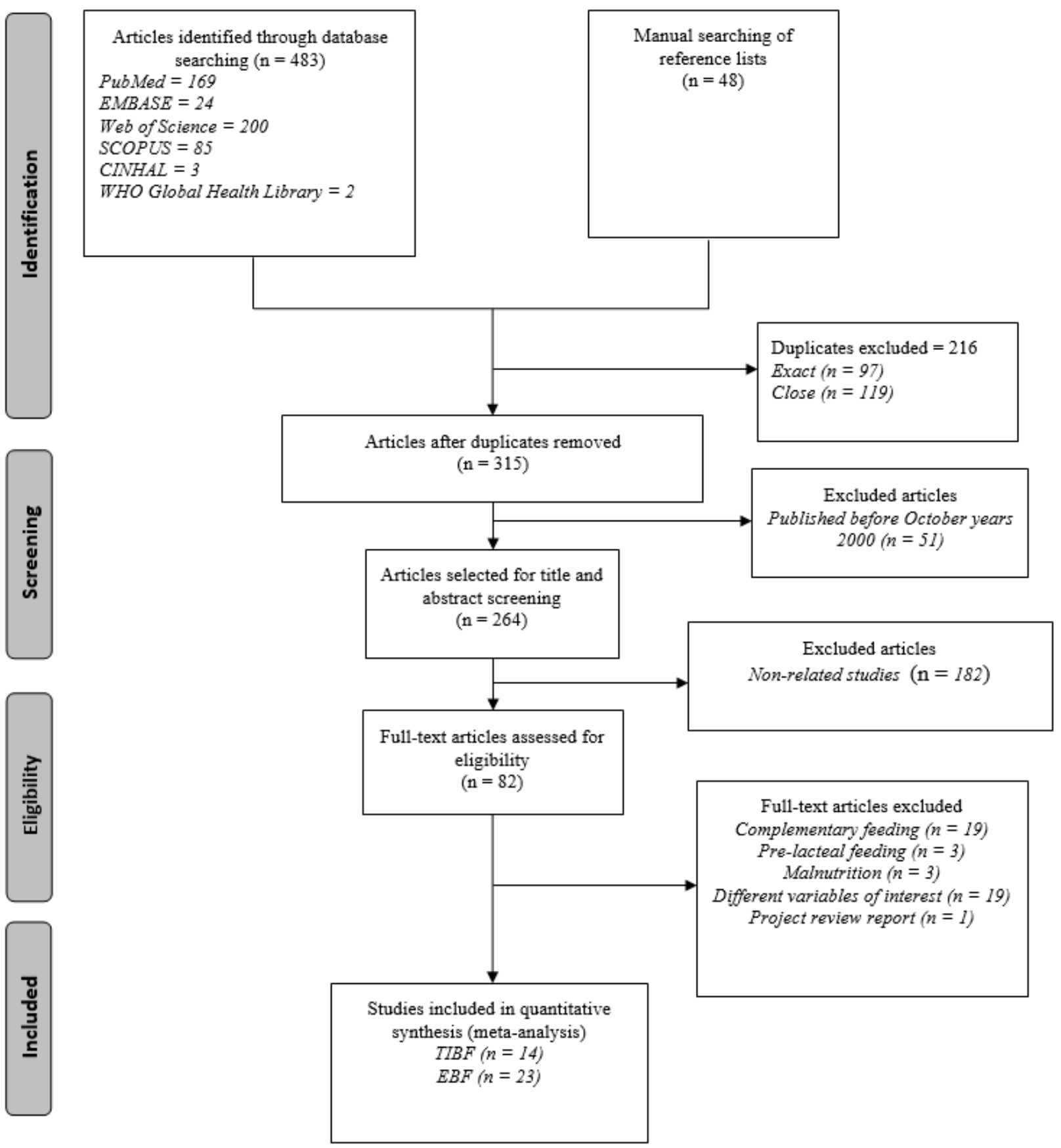

Figure 1: PRISMA flow diagram of literature screening and selection process; " $n$ " in each stage represents the total number of studies that fulfilled a particular criterion. 


\section{Study characteristics}

Of these 14 studies on TIBF, most studies were conducted in Southern Nations Nationalities and People (SNNPR) region and Oromia region. Regarding maternal/caregiver's residence, six and three studies conducted in urban and rural dwellers respectively (Table 1).

Table 1: Characteristics of studies included on TIBF

\begin{tabular}{|c|c|c|c|c|c|c|c|c|}
\hline \multirow{2}{*}{$\begin{array}{l}\text { Au- } \\
\text { thor/publicati } \\
\text { on year }\end{array}$} & \multirow{2}{*}{$\begin{array}{l}\text { Study area } \\
\text { (Region and } \\
\text { place) }\end{array}$} & \multirow{2}{*}{$\begin{array}{l}\text { Study } \\
\text { meth- } \\
\text { od/desig } \\
\text { n }\end{array}$} & \multirow[t]{2}{*}{ Study population } & \multirow[t]{2}{*}{$\begin{array}{l}\text { Calculated Sample } \\
\text { size/Participated }\end{array}$} & \multirow[t]{2}{*}{ Factors } & \multicolumn{3}{|c|}{$\begin{array}{c}\text { Breastfeeding initiation } \\
\text { (outcome) }\end{array}$} \\
\hline & & & & & & $\begin{array}{l}\text { Within } \\
1 \text { hour }\end{array}$ & $\begin{array}{l}\text { After } 1 \\
\text { hour }\end{array}$ & Total \\
\hline \multicolumn{9}{|c|}{ Maternal/Caregiver's age versus timely initiation of breastfeeding } \\
\hline \multirow{3}{*}{$\begin{array}{l}\text { Wolde et.al. } \\
2014\end{array}$} & \multirow{3}{*}{$\begin{array}{l}\text { Oromia, } \\
\text { Nekemte twon }\end{array}$} & \multirow{3}{*}{$\begin{array}{l}\text { Cross- } \\
\text { sectional }\end{array}$} & \multirow{3}{*}{$\begin{array}{l}\text { Mothers who had } \\
\text { child less than } 24 \\
\text { month }\end{array}$} & \multirow[t]{3}{*}{$182 / 174$} & $<25$ years & 43 & 5 & 48 \\
\hline & & & & & $\geq 25$ years & 111 & 15 & 126 \\
\hline & & & & & Total & 154 & 20 & 174 \\
\hline \multirow{3}{*}{$\begin{array}{l}\text { Woldemichael } \\
\text { et.al. } 2016\end{array}$} & \multirow{3}{*}{$\begin{array}{l}\text { Oromia, Tiyo } \\
\text { Woreda }\end{array}$} & \multirow{3}{*}{$\begin{array}{l}\text { Cross- } \\
\text { sectional } \\
\text { study }\end{array}$} & \multirow{3}{*}{$\begin{array}{l}\text { mothers who have } \\
\text { children Less Than } \\
\text { One Year Age }\end{array}$} & \multirow[t]{3}{*}{$386 / 373$} & $<25$ years & 83 & 39 & 122 \\
\hline & & & & & $\geq 25$ years & 168 & 83 & 251 \\
\hline & & & & & Total & 251 & 122 & 373 \\
\hline \multirow{3}{*}{$\begin{array}{l}\text { Adugna et.al } \\
2014\end{array}$} & \multirow{3}{*}{$\begin{array}{l}\text { SNNPR, Arba } \\
\text { Minch Zuria }\end{array}$} & \multirow{3}{*}{$\begin{array}{l}\text { cross- } \\
\text { sectional } \\
\text { study }\end{array}$} & \multirow{3}{*}{$\begin{array}{l}\text { Women who had } \\
\text { children under two } \\
\text { years }\end{array}$} & \multirow[t]{3}{*}{$384 / 383$} & $<25$ years & 181 & 132 & 313 \\
\hline & & & & & $\geq 25$ years & 38 & 32 & 70 \\
\hline & & & & & Total & 219 & 164 & 383 \\
\hline \multirow{3}{*}{$\begin{array}{l}\text { Beyene et.al } \\
2017\end{array}$} & \multirow{3}{*}{$\begin{array}{l}\text { SNNPR, Dale } \\
\text { Woreda }\end{array}$} & & mothers of children & $634 / 634$ & $<25$ years & 180 & 49 & 229 \\
\hline & & sectional & under 24 months & & $\geq 25$ years & 337 & 52 & 389 \\
\hline & & & & & Total & 517 & 101 & 618 \\
\hline Alemayehu & Tigray, Axum & cross & mothers who had & $418 / 418$ & $<25$ years & 75 & 49 & 124 \\
\hline et.al 2014 & town & sectional & children aged 6-12 & & $\geq 25$ years & 169 & 125 & 294 \\
\hline & & study & months & & Total & 244 & 174 & 418 \\
\hline Berhe et.al & Tigray, & Cross- & mothers of children & $361 / 361$ & $<25$ years & 120 & 27 & 147 \\
\hline 2013 & Mekelle town & sectional & aged 0 to 24 months & & $\geq 25$ years & 158 & 52 & 210 \\
\hline & & study & & & Total & 278 & 79 & 357 \\
\hline Setegn et.al & Oromia, Goba & cross & mothers with children & $668 / 608$ & $<25$ years & 107 & 108 & 215 \\
\hline 2011 & district & sectional & (<12 months & & $\geq 25$ years & 207 & 177 & 384 \\
\hline & & study & & & Total & 314 & 285 & 599 \\
\hline Tamiru et.al & SNNPR, Arba & cross- & mothers of infants & $384 / 384$ & $<25$ years & 150 & 109 & 259 \\
\hline 2015 & Minch Zuria & sectional & aged two years and & & $\geq 25$ years & 70 & 54 & 124 \\
\hline & Woreda & study & younger & & Total & 220 & 163 & 383 \\
\hline Regassa 2014 & SNNPR, & Cross- & with infants aged be- & $1100 / 1094$ & $<25$ years & 354 & 77 & 431 \\
\hline & Sidama zone & sectional & tween 0 and 6 & & $\geq 25$ years & 522 & 141 & 663 \\
\hline & & study & months old & & Total & 876 & 218 & 1094 \\
\hline Ekubay et al & Addis Ababa & Cross- & mothers with infants & $597 / 583$ & $<25$ years & 134 & 94 & 228 \\
\hline 2018 & Town & sectional & younger than or equal & & $\geq 25$ years & 195 & 141 & 336 \\
\hline & & study & to six months of age & & Total & 329 & 235 & 564 \\
\hline Discarding col & trum versus tin & ely initiatic & 1 of breastfeeding & & & & & \\
\hline Wolde et.al. & Oromia, & Cross- & mothers who had & $182 / 174$ & Discarding & 10 & 3 & 13 \\
\hline 2014 & Nekemte town & $\begin{array}{l}\text { sectional } \\
\text { study }\end{array}$ & $\begin{array}{l}\text { child less than } 24 \\
\text { month }\end{array}$ & & No & 144 & 17 & 161 \\
\hline & & & & & Total & 154 & 20 & 174 \\
\hline Adugna et.al. & SNNPR, & Cross- & Mothers with infants & $541 / 529$ & Discarding & 21 & 21 & 42 \\
\hline 2014 & Hawassa city & & aged 0-6 months & & No & 198 & 143 & 341 \\
\hline
\end{tabular}




\begin{tabular}{|c|c|c|c|c|c|c|c|c|}
\hline & & study & & & Total & 219 & 164 & 383 \\
\hline \multirow{3}{*}{$\begin{array}{l}\text { Hailemariam } \\
\text { et.al. } 2015\end{array}$} & \multirow{3}{*}{$\begin{array}{l}\text { Oromia, East } \\
\text { Wollega zone }\end{array}$} & \multirow{3}{*}{$\begin{array}{l}\text { cross- } \\
\text { sectional } \\
\text { study }\end{array}$} & \multirow{3}{*}{$\begin{array}{l}\text { Mothers who had } \\
\text { children less than } 24 \\
\text { months }\end{array}$} & \multirow[t]{3}{*}{$594 / 593$} & Discarding & 30 & 15 & 45 \\
\hline & & & & & No & 443 & 81 & 524 \\
\hline & & & & & Total & 473 & 96 & 569 \\
\hline \multirow[t]{3}{*}{ Tewabe 2016} & \multirow{3}{*}{$\begin{array}{l}\text { Amhara, Mot- } \\
\text { ta town }\end{array}$} & \multirow{3}{*}{$\begin{array}{l}\text { cross- } \\
\text { sectional } \\
\text { study }\end{array}$} & \multirow{3}{*}{$\begin{array}{l}\text { mothers with infant } \\
\text { less than six month } \\
\text { old }\end{array}$} & \multirow[t]{3}{*}{$423 / 405$} & Discarding & 49 & 33 & 82 \\
\hline & & & & & No & 270 & 53 & 323 \\
\hline & & & & & Total & 319 & 86 & 405 \\
\hline \multirow{3}{*}{$\begin{array}{l}\text { Tilahun et.al. } \\
2016\end{array}$} & \multirow{3}{*}{$\begin{array}{l}\text { Amhara, } \\
\text { Debre Berhan } \\
\text { town }\end{array}$} & \multirow{3}{*}{$\begin{array}{l}\text { cross } \\
\text { sectional } \\
\text { study }\end{array}$} & \multirow{3}{*}{$\begin{array}{l}\text { mothers who had } \\
\text { children less than six } \\
\text { months of age }\end{array}$} & \multirow[t]{3}{*}{$416 / 409$} & Discarding & 15 & 46 & 61 \\
\hline & & & & & No & 241 & 91 & 332 \\
\hline & & & & & Total & 256 & 137 & 393 \\
\hline \multirow{3}{*}{$\begin{array}{l}\text { Liben and } \\
\text { Yesuf } 2016\end{array}$} & \multirow{3}{*}{$\begin{array}{l}\text { Afar, Amibara } \\
\text { district }\end{array}$} & \multirow{3}{*}{$\begin{array}{l}\text { Cross- } \\
\text { sectional } \\
\text { study }\end{array}$} & \multirow{3}{*}{$\begin{array}{l}\text { mothers of children } \\
\text { aged } \\
\text { less than } 24 \text { months }\end{array}$} & \multirow[t]{3}{*}{$407 / 403$} & Discarding & 83 & 142 & 225 \\
\hline & & & & & No & 68 & 88 & 156 \\
\hline & & & & & Total & 151 & 230 & 381 \\
\hline
\end{tabular}

Majority of studies on EBF were done in Amhara, SNNP and Oromia regions with 7, 6 and 3 studies respectively. Likewise, nine and seven studies conducted in urban and rural dwellers respectively. Furthermore, two studies used a nationally representative data of Ethiopian

Demographic and Health Survey (EDHS) (Table 2).

Table 2: Characteristics of studies included on EBF

\begin{tabular}{|c|c|c|c|c|c|c|c|c|}
\hline \multirow{2}{*}{$\begin{array}{l}\text { Au- } \\
\text { thor/publica } \\
\text { tion year }\end{array}$} & \multirow{2}{*}{$\begin{array}{l}\text { Study area } \\
\text { (Region and } \\
\text { place) }\end{array}$} & \multirow{2}{*}{$\begin{array}{l}\text { Study } \\
\text { meth- } \\
\text { od/design }\end{array}$} & \multirow[t]{2}{*}{ Study population } & \multirow{2}{*}{$\begin{array}{l}\text { Calculated Sample } \\
\text { size/Participated }\end{array}$} & \multirow[t]{2}{*}{ Factors } & \multicolumn{3}{|c|}{ Exclusive breastfeeding } \\
\hline & & & & & & Yes & No & Total \\
\hline \multicolumn{9}{|c|}{ Maternal/Caregiver's age versus exclusive breastfeeding } \\
\hline \multirow[t]{3}{*}{ Abera 2012} & \multirow{3}{*}{$\begin{array}{l}\text { Harari, Harar } \\
\text { twon }\end{array}$} & \multirow{3}{*}{$\begin{array}{l}\text { Cross- } \\
\text { sectional } \\
\text { study }\end{array}$} & \multirow{3}{*}{$\begin{array}{l}\text { Mothers of children } \\
\text { aged less than two years }\end{array}$} & \multirow[t]{3}{*}{$604 / 583$} & $<25$ years & 49 & 31 & 80 \\
\hline & & & & & $\geq 25$ years & 158 & 161 & 319 \\
\hline & & & & & Total & 207 & 192 & 399 \\
\hline \multirow{3}{*}{$\begin{array}{l}\text { Getahun } \\
\text { et.al. } 2017\end{array}$} & \multirow{3}{*}{$\begin{array}{l}\text { SNNPR, Kemba } \\
\text { Woreda }\end{array}$} & \multirow{3}{*}{$\begin{array}{l}\text { Cross- } \\
\text { sectional } \\
\text { study }\end{array}$} & \multirow{3}{*}{$\begin{array}{l}\text { Mothers who have chil- } \\
\text { dren from } 6 \text { months to } \\
2 \text { years age }\end{array}$} & \multirow[t]{3}{*}{$567 / 562$} & $<25$ years & 134 & 105 & 239 \\
\hline & & & & & $\geq 25$ years & 200 & 123 & 323 \\
\hline & & & & & Total & 334 & 228 & 562 \\
\hline \multirow{3}{*}{$\begin{array}{l}\text { Asfaw et.al. } \\
2015\end{array}$} & \multirow{3}{*}{$\begin{array}{l}\text { Amhara, Debre } \\
\text { Berhan } \\
\text { District }\end{array}$} & \multirow{3}{*}{$\begin{array}{l}\text { Cross sec- } \\
\text { tional study }\end{array}$} & \multirow{3}{*}{$\begin{array}{l}\text { Mothers with their index } \\
\text { infant aged under } 12 \\
\text { months }\end{array}$} & \multirow[t]{3}{*}{$634 / 634$} & $<25$ years & 47 & 61 & 108 \\
\hline & & & & & $\geq 25$ years & 388 & 138 & 526 \\
\hline & & & & & Total & 435 & 199 & 634 \\
\hline \multirow{3}{*}{$\begin{array}{l}\text { Gizaw et.al. } \\
2017\end{array}$} & \multirow{3}{*}{$\begin{array}{l}\text { Afar, } \\
\text { Hadaleala dis- } \\
\text { trict }\end{array}$} & \multirow{3}{*}{$\begin{array}{l}\text { Cross- } \\
\text { sectional } \\
\text { study }\end{array}$} & \multirow{3}{*}{$\begin{array}{l}\text { Mothers who have chil- } \\
\text { dren aged between } 6 \text { and } \\
24 \text { months }\end{array}$} & \multirow[t]{3}{*}{$258 / 254$} & $<25$ years & 56 & 23 & 79 \\
\hline & & & & & $\geq 25$ years & 132 & 43 & 175 \\
\hline & & & & & Total & 188 & 66 & 254 \\
\hline \multirow{3}{*}{$\begin{array}{l}\text { Hunegnaw } \\
\text { et.al. } 2017\end{array}$} & \multirow{3}{*}{$\begin{array}{l}\text { Amhara, } \\
\text { Gozamin dis- } \\
\text { trict }\end{array}$} & \multirow{3}{*}{$\begin{array}{l}\text { Cross- } \\
\text { sectional } \\
\text { study }\end{array}$} & \multirow{3}{*}{$\begin{array}{l}\text { Mothers who had In- } \\
\text { fants aged between } 6 \\
\text { and } 12 \text { months }\end{array}$} & \multirow[t]{3}{*}{$506 / 478$} & $<25$ years & 72 & 26 & 98 \\
\hline & & & & & $\geq 25$ years & 286 & 104 & 390 \\
\hline & & & & & Total & 358 & 130 & 488 \\
\hline & SNNPR, Offa & Cross- & Mothers of infants & $403 / 396$ & $<25$ years & 96 & 22 & 118 \\
\hline 2016 & district & sectional & younger than 6 months & & $\geq 25$ years & 213 & 65 & 278 \\
\hline & & study & & & Total & 309 & 87 & 396 \\
\hline Setegn et.al. & Oromia, Bale & Cross- & Mothers-infant pairs & $668 / 608$ & $<25$ years & 79 & 27 & 106 \\
\hline 2012 & Zone, Goba & sectional & & & $\geq 25$ years & 120 & 53 & 173 \\
\hline
\end{tabular}




\begin{tabular}{|c|c|c|c|c|c|c|c|c|}
\hline & district & study & & & Total & 199 & 80 & 279 \\
\hline \multirow{3}{*}{$\begin{array}{l}\text { Sonko et.al. } \\
2015\end{array}$} & \multirow{3}{*}{$\begin{array}{l}\text { SNNPR, } \\
\text { Halaba special } \\
\text { woreda }\end{array}$} & \multirow{3}{*}{$\begin{array}{l}\text { Cross- } \\
\text { sectional } \\
\text { study }\end{array}$} & \multirow{3}{*}{$\begin{array}{l}\text { Mothers } \\
\text { With children less than } \\
\text { six months of age }\end{array}$} & \multirow[t]{3}{*}{$422 / 420$} & $<25$ years & 56 & 24 & 80 \\
\hline & & & & & $\geq 25$ years & 240 & 100 & 340 \\
\hline & & & & & Total & 296 & 124 & 420 \\
\hline \multirow{3}{*}{$\begin{array}{l}\text { Regassa. } \\
2014\end{array}$} & \multirow{3}{*}{$\begin{array}{l}\text { SNNPR, } \\
\text { Sidama zone }\end{array}$} & \multirow{3}{*}{$\begin{array}{l}\text { Cross- } \\
\text { sectional } \\
\text { study }\end{array}$} & \multirow{3}{*}{$\begin{array}{l}\text { with infants aged be- } \\
\text { tween } 0 \text { and } 6 \text { months } \\
\text { old }\end{array}$} & \multirow[t]{3}{*}{$1100 / 1094$} & $<25$ years & 78 & 14 & 92 \\
\hline & & & & & $\geq 25$ years & 120 & 22 & 142 \\
\hline & & & & & Total & 198 & 36 & 234 \\
\hline \multirow{3}{*}{$\begin{array}{l}\text { Alemayehu } \\
\text { et.al. } 2014\end{array}$} & \multirow{3}{*}{$\begin{array}{l}\text { Tigray, Axum } \\
\text { town }\end{array}$} & \multirow{3}{*}{$\begin{array}{l}\text { cross sec- } \\
\text { tional study }\end{array}$} & \multirow{3}{*}{$\begin{array}{l}\text { mothers who had chil- } \\
\text { dren aged 6-12 } \\
\text { months }\end{array}$} & \multirow[t]{3}{*}{$418 / 418$} & $<25$ years & 46 & 78 & 124 \\
\hline & & & & & $\geq 25$ years & 125 & 169 & 294 \\
\hline & & & & & Total & 171 & 247 & 418 \\
\hline \multirow{3}{*}{$\begin{array}{l}\text { Berhe et.al. } \\
2013\end{array}$} & \multirow{3}{*}{$\begin{array}{l}\text { Tigray, } \\
\text { Mekelle town }\end{array}$} & \multirow{3}{*}{$\begin{array}{l}\text { Cross- } \\
\text { sectional } \\
\text { study }\end{array}$} & \multirow{3}{*}{$\begin{array}{l}\text { mothers of children aged } \\
0 \text { to } 24 \text { months }\end{array}$} & \multirow[t]{3}{*}{$361 / 361$} & $<25$ years & 54 & 32 & 86 \\
\hline & & & & & $\geq 25$ years & 56 & 39 & 95 \\
\hline & & & & & Total & 110 & 71 & 181 \\
\hline Teka et al. & Tigray, Enderta & & Mothers having children & $541 / 530$ & $<25$ years & 139 & 52 & 191 \\
\hline 2015 & Woreda & sectional & aged less than 24 & & $\geq 25$ years & 233 & 106 & 339 \\
\hline & & & & & Total & 372 & 158 & 530 \\
\hline Newborn age & versus exclusive $\mathbf{b}$ & reastfeeding & & & & & & \\
\hline Arage et.al. & Amhara, Debre & Cross- & Mothers of Infants Less & $470 / 453$ & $\leq 3$ months & 201 & 80 & 281 \\
\hline 2016 & Tabor Town & sectional & Than Six Months of Age & & $>3$ months & 96 & 72 & 168 \\
\hline & & study & & & Total & 297 & 152 & 449 \\
\hline Alemayehu & 9 regions, $\mathrm{Na}-$ & & Women with infants & $14,500 / 1142$ & $\leq 3$ months & 682 & 1335 & 2017 \\
\hline et.al. 2009 & tional & demograph- & Less than six months of & & $>3$ months & 326 & 683 & 1009 \\
\hline & & & & & Total & 1008 & 2018 & 3026 \\
\hline Asemahagn. & Amhara, Azezo & Cross- & Women having children & $346 / 332$ & $\leq 3$ months & 129 & 22 & 151 \\
\hline 2016 & district & sectional & aged from $0-6$ months & & $>3$ months & 133 & 48 & 181 \\
\hline & & study & & & Total & 262 & 70 & 332 \\
\hline Liben et.al. & Afar, Dubti & & Mothers of infants aged & $346 / 333$ & $\leq 3$ months & 199 & 36 & 235 \\
\hline 2016 & & sectional & less than 6 months & & $>3$ months & 71 & 27 & 98 \\
\hline & & & & & Total & 270 & 63 & 333 \\
\hline Seid et.al. & Amhara, Bahir & Cross- & Mothers who & $819 / 819$ & $\leq 3$ months & 103 & 91 & 194 \\
\hline 2013 & Dar city & sectional & Delivered in the last 12 & & $>3$ months & 300 & 366 & 666 \\
\hline & & study & months & & Total & 403 & 457 & 860 \\
\hline Setegn et.al. & & & Mothers-infant pairs & $668 / 608$ & $\leq 3$ months & 122 & 27 & 149 \\
\hline 2012 & Zone, Goba & sectional & & & $>3$ months & 61 & 33 & 94 \\
\hline & & & & & Total & 183 & 60 & 243 \\
\hline Sonko et.al. & SNNPR, & Cross- & Mothers & $422 / 420$ & $\leq 3$ months & 121 & 43 & 164 \\
\hline 2015 & Halaba special & sectional & With children less than & & $>3$ months & 175 & 81 & 256 \\
\hline & woreda & study & six months of age & & Total & 296 & 124 & 420 \\
\hline Tadesse & SNNPR, Sorro & Cross- & Mothers With infants & $602 / 579$ & $\leq 3$ months & 214 & 129 & 343 \\
\hline et.al. 2016 & District & sectional & aged of $0-5$ months & & $>3$ months & 56 & 115 & 171 \\
\hline & & Study & & & Total & 270 & 244 & 514 \\
\hline Tewabe & Amhara, Motta & Cross- & Mothers with infant less & $423 / 405$ & $\leq 3$ months & 106 & 68 & 174 \\
\hline et.al. 2017 & town, East & sectional & than six Months old & & $>3$ months & 97 & 134 & 231 \\
\hline & Gojjam zone & Study & & & Total & 203 & 202 & 405 \\
\hline Berhe et.al. & Tigray, & Cross- & mothers of children aged & $361 / 361$ & $\leq 3$ months & 96 & 51 & 147 \\
\hline 2013 & Mekelle town & sectional & 0 to 24 months & & $>3$ months & 14 & 20 & 34 \\
\hline & & study & & & Total & 110 & 71 & 181 \\
\hline Discarding C & ostrum versus exc & usive breastf & eding & & & & & \\
\hline Arage et.al & Amhara, Debre & Cross- & Mothers of Infants Less & $470 / 453$ & Discarding & 7 & 5 & 12 \\
\hline 2016 & Tabor Town & sectional & Than Six Months of Age & & No & 361 & 280 & 641 \\
\hline
\end{tabular}




\begin{tabular}{|c|c|c|c|c|c|c|c|c|}
\hline & & study & & & Total & 368 & 285 & 653 \\
\hline \multirow{3}{*}{$\begin{array}{l}\text { Egata et.al. } \\
2013\end{array}$} & \multirow{3}{*}{$\begin{array}{l}\text { Oromia, Kersa } \\
\text { district }\end{array}$} & \multirow{3}{*}{$\begin{array}{l}\text { Cross- } \\
\text { sectional } \\
\text { study (DHS } \\
\text { based) }\end{array}$} & \multirow{3}{*}{$\begin{array}{l}\text { Mothers of children } \\
\text { under-two years of age }\end{array}$} & \multirow[t]{3}{*}{$881 / 860$} & Discarding & 44 & 29 & 73 \\
\hline & & & & & No & 573 & 214 & 787 \\
\hline & & & & & Total & 617 & 243 & 860 \\
\hline \multirow{3}{*}{$\begin{array}{l}\text { Lenja et.al. } \\
2016\end{array}$} & \multirow{3}{*}{$\begin{array}{l}\text { SNNPR, Offa } \\
\text { district }\end{array}$} & \multirow{3}{*}{$\begin{array}{l}\text { Cross- } \\
\text { sectional } \\
\text { study }\end{array}$} & \multirow{3}{*}{$\begin{array}{l}\text { Mothers of infants } \\
\text { younger than } 6 \text { months }\end{array}$} & \multirow[t]{3}{*}{$403 / 396$} & Discarding & 53 & 33 & 86 \\
\hline & & & & & No & 256 & 49 & 305 \\
\hline & & & & & Total & 309 & 82 & 391 \\
\hline \multirow{3}{*}{$\begin{array}{l}\text { Liben et.al. } \\
2016\end{array}$} & \multirow{3}{*}{$\begin{array}{l}\text { Afar, Dubti } \\
\text { town }\end{array}$} & \multirow{3}{*}{$\begin{array}{l}\text { Cross- } \\
\text { sectional } \\
\text { study }\end{array}$} & \multirow{3}{*}{$\begin{array}{l}\text { Mothers of infants aged } \\
\text { less than } 6 \text { months }\end{array}$} & \multirow[t]{3}{*}{$346 / 333$} & Discarding & 33 & 19 & 52 \\
\hline & & & & & No & 237 & 44 & 281 \\
\hline & & & & & Total & 270 & 63 & 333 \\
\hline \multirow{3}{*}{$\begin{array}{l}\text { Mekuria } \\
\text { et.al. } 2015\end{array}$} & \multirow{3}{*}{$\begin{array}{l}\text { Amhara, Debre } \\
\text { Markos }\end{array}$} & \multirow{3}{*}{$\begin{array}{l}\text { Cross- } \\
\text { sectional } \\
\text { study }\end{array}$} & \multirow{3}{*}{$\begin{array}{l}\text { Mothers who had an } \\
\text { infant Less than six } \\
\text { months old }\end{array}$} & \multirow[t]{3}{*}{$423 / 413$} & Discarding & 83 & 71 & 154 \\
\hline & & & & & No & 168 & 91 & 259 \\
\hline & & & & & Total & 251 & 162 & 413 \\
\hline \multirow{3}{*}{$\begin{array}{l}\text { Seid et.al. } \\
2013\end{array}$} & \multirow{3}{*}{$\begin{array}{l}\text { Amhara, Bahir } \\
\text { Dar city }\end{array}$} & \multirow{3}{*}{$\begin{array}{l}\text { Cross- } \\
\text { sectional } \\
\text { study }\end{array}$} & \multirow{3}{*}{$\begin{array}{l}\text { Mothers who } \\
\text { Delivered in the last } 12 \\
\text { months }\end{array}$} & \multirow[t]{3}{*}{$819 / 819$} & Discarding & 56 & 80 & 136 \\
\hline & & & & & No & 356 & 323 & 679 \\
\hline & & & & & Total & 412 & 403 & 815 \\
\hline \multirow{3}{*}{$\begin{array}{l}\text { Tadesse } \\
\text { et.al. } 2016\end{array}$} & \multirow{3}{*}{$\begin{array}{l}\text { SNNPR, Sorro } \\
\text { District }\end{array}$} & \multirow{3}{*}{$\begin{array}{l}\text { Cross- } \\
\text { sectional } \\
\text { Study }\end{array}$} & Mothers With infants & $602 / 579$ & Discarding & 68 & 101 & 169 \\
\hline & & & aged of $0-5$ months & & No & 202 & 143 & 345 \\
\hline & & & & & Total & 270 & 244 & 514 \\
\hline Tewabe & Amhara, Motta & Cross- & Mothers with infant less & $423 / 405$ & Discarding & 18 & 64 & 82 \\
\hline et.al. 2017 & town, East & sectional & than six Months old & & No & 185 & 138 & 323 \\
\hline & Gojjam zone & Study & & & Total & 203 & 202 & 405 \\
\hline Tamiru et.al. & Oromia, Jimma & Cross- & Mothers of index chil- & $384 / 382$ & Discarding & 61 & 42 & 103 \\
\hline 2012 & Arjo Woreda & sectional & dren aged & & No & 122 & 157 & 279 \\
\hline & & study & 0 to 6 months & & Total & 183 & 199 & 382 \\
\hline Tamiru et.al. & SNNPR, Arba & cross- & mothers of infants aged & $384 / 384$ & Discarding & 23 & 19 & 42 \\
\hline 2015 & Minch Zuria & sectional & two years and younger & & No & 232 & 110 & 342 \\
\hline & Woreda & study & & & Total & 255 & 129 & 384 \\
\hline Alemayehu & Tigray, Axum & cross sec- & mothers who had chil- & $418 / 418$ & Discarding & 49 & 118 & 167 \\
\hline et.al. 2014 & town & tional study & dren aged 6-12 & & No & 122 & 66 & 188 \\
\hline & & & months & & Total & 171 & 184 & 355 \\
\hline Teka et al. & Tigray, & Cross- & Mothers having chil- & $541 / 530$ & Discarding & 350 & 141 & 491 \\
\hline 2015 & Enderta & sectional & dren aged less than & & No & 22 & 17 & 39 \\
\hline & Woreda & study & 24 months & & Total & 372 & 158 & 530 \\
\hline
\end{tabular}

\section{Timely initiation of breastfeeding (TIBF)}

Among 14 studies, 10 studies ${ }^{(46-55)}$ reported the association between TIBF and maternal/caregiver's age in 4,963 mothers. The pooled odds ratio (OR) of maternal/caregiver's age was 0.98 (95\% CI $0.83-1.15, \mathrm{p}=0.78$ ) (figure 2 ). Although not statistically significant, mothers $\geq 25$ years old age had $2 \%$ lower chance of initiating breastfeeding within one hour compared to their younger counterparts. Egger's regression test for funnel plot asymmetry was not significant $(z=-0.40, p=0.69)$. 


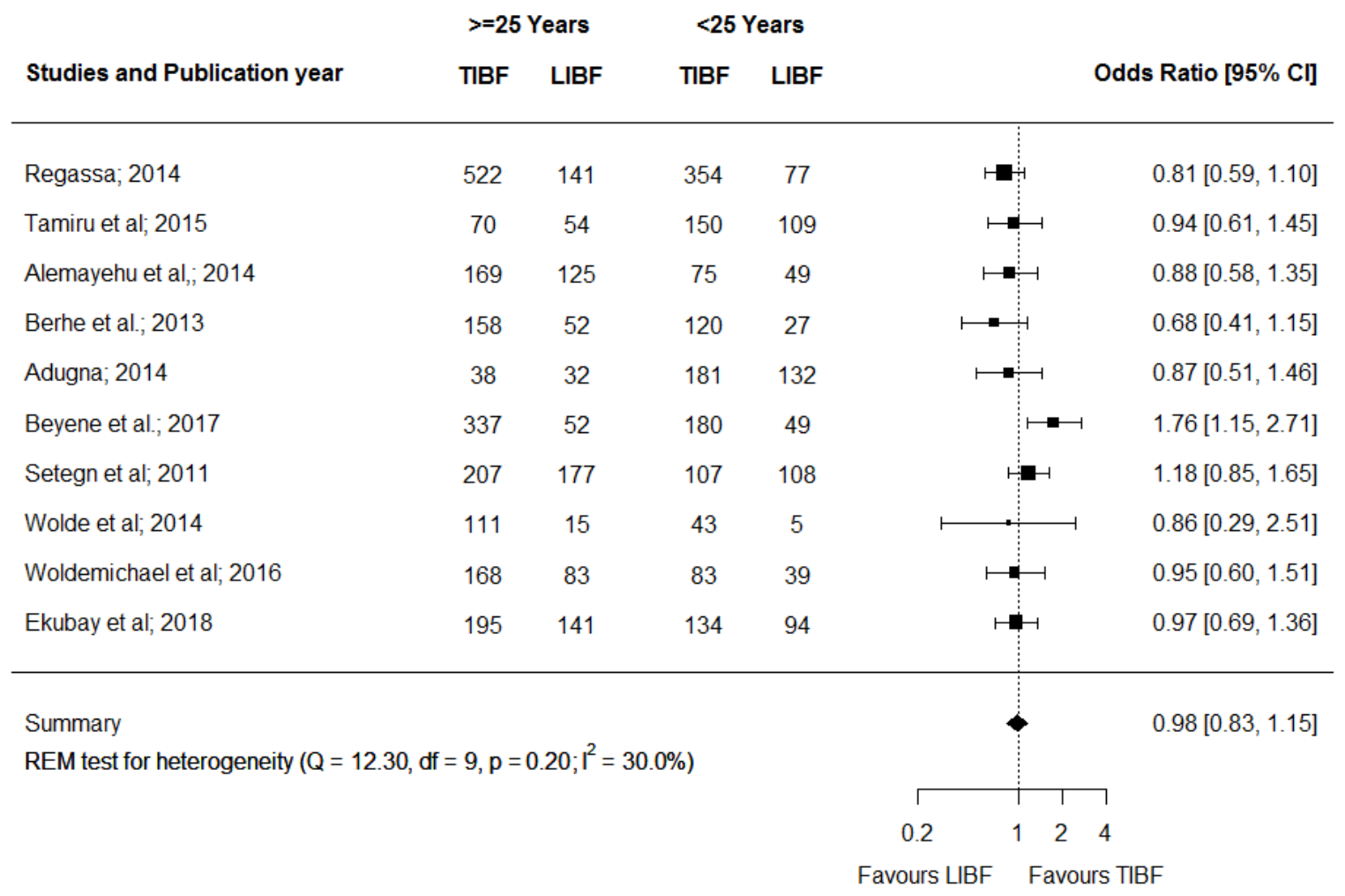

Figure 2:Forest plot of the unadjusted odds ratios with corresponding 95\% CIs of studies on the association of maternal/caregiver's age and TIBF. The horizontal line represents the confidence interval, the box and its size in the middle of the horizontal line represent the weight of sample size. The polygon represents the pooled odds ratio. TIBF = timely initiation of breastfeeding; LIBF = late initiation of breastfeeding; REM = random-effects model.

Likewise, 6 out of 14 studies reported the association between TIBF and colostrum discarding in 2,305 mothers $(46,48,56-59)$. The pooled OR of colostrum discarding was found to be $0.38(95 \% \mathrm{CI}$ $0.21-0.68, p=0.001$ ) (figure 3). Compared to mothers who feed colostrum, mothers who discard colostrum had $62 \%$ significantly lower chance of initiating breastfeeding within one hour. Egger's regression test for funnel plot asymmetry was not significant $(z=-0.24, p=0.81)$. 


\section{D colostrum NotD colostrum}

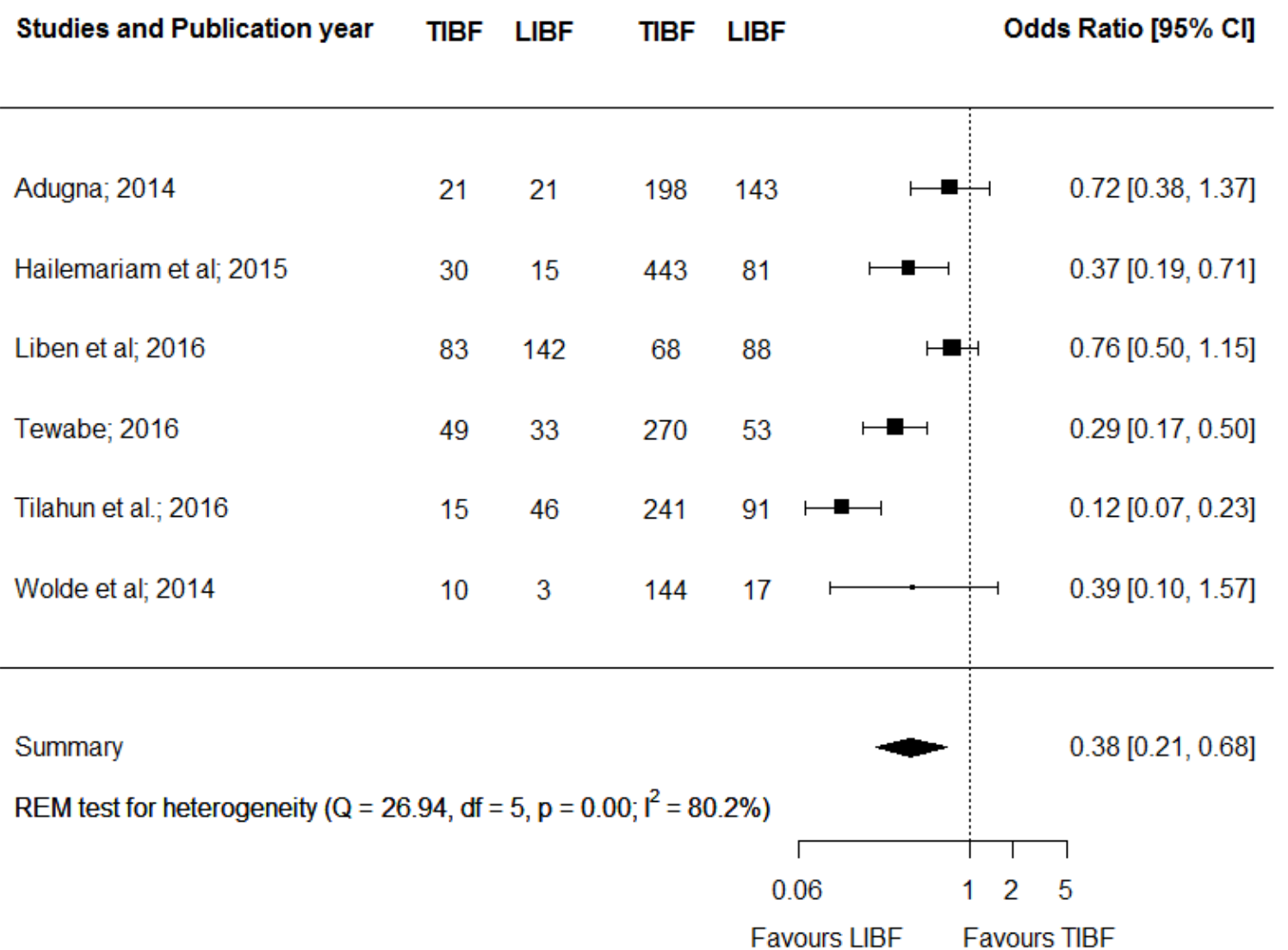

Figure 3: Forest plot of the unadjusted odds ratios with corresponding 95\% CIs of studies on the association of colostrum discarding and TIBF. The horizontal line represents the confidence interval, the box and its size in the middle of the horizontal line represent the weight of sample size. The polygon represents the pooled odds ratio. TIBF = timely initiation of breastfeeding; $\mathrm{LIBF}=$ late initiation of breastfeeding; REM = random-effects model; $\mathrm{D}=$ Discarding; NotD = Not discarding.

\section{Exclusive breastfeeding}

Twelve studies ${ }^{(50,51,54,60-68)}$ involving 4,929 individuals reported the association between EBF and maternal/caregiver's age. As showed in figure 4, the pooled OR of maternal/caregiver's age was $1.07(95 \%$ CI $0.81-1.40, \mathrm{p}=0.63)$. Mothers $\geq 25$ years old age had $7 \%$ higher chance of exclusively breastfeeding during the first six months compared to mothers $<25$ years old; 
however, it was not statistically significant. Egger's regression test for funnel plot asymmetry was not significant $(\mathrm{z}=-0.99, \mathrm{p}=0.32)$.

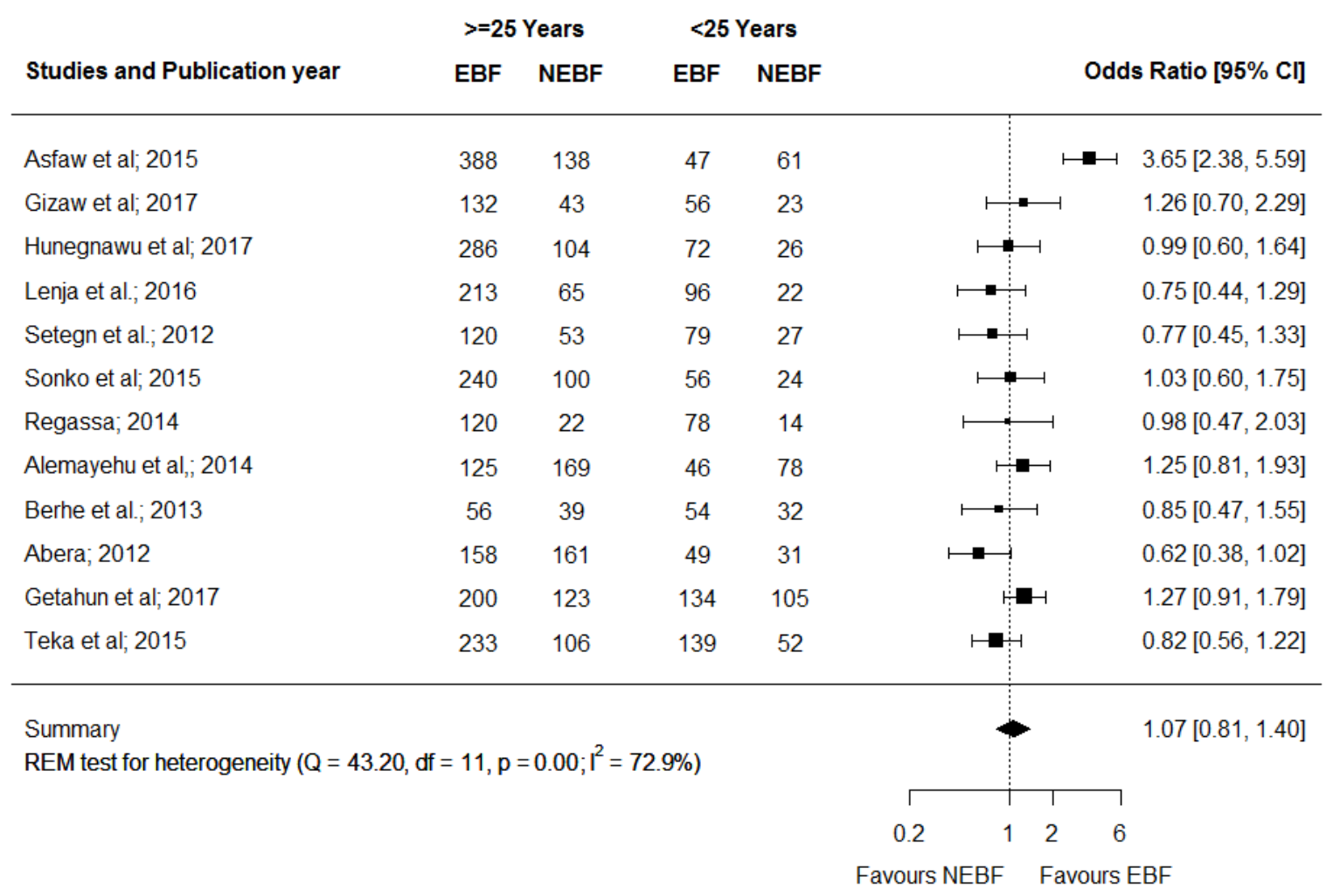

Figure 4: Forest plot of the unadjusted odds ratios with corresponding 95\% CIs of studies on the association of maternal/caregiver's age and EBF. The horizontal line represents the confidence interval, the box and its size in the middle of the horizontal line represent the weight of sample size. The polygon represents the pooled odds ratio. EBF = Exclusive breastfeeding; NEBF = Non-exclusive of breastfeeding; REM = random-effects model.

In addition, ten ${ }^{(51,65,66,69-75)}$ out of 23 studies reported the association between EBF and infant age with a total sample of 6,763 mothers. The pooled OR of infant age was 1.86 (95\% CI 1.45 $2.39, \mathrm{p}<0.001$ ) (figure 5). Children $\leq 3$ months old had $86 \%$ statistically significant higher chance of being exclusively breastfed compared to children older than 3 months. Egger's regression test for funnel plot asymmetry was not significant $(z=2.31, p=0.02)$. 


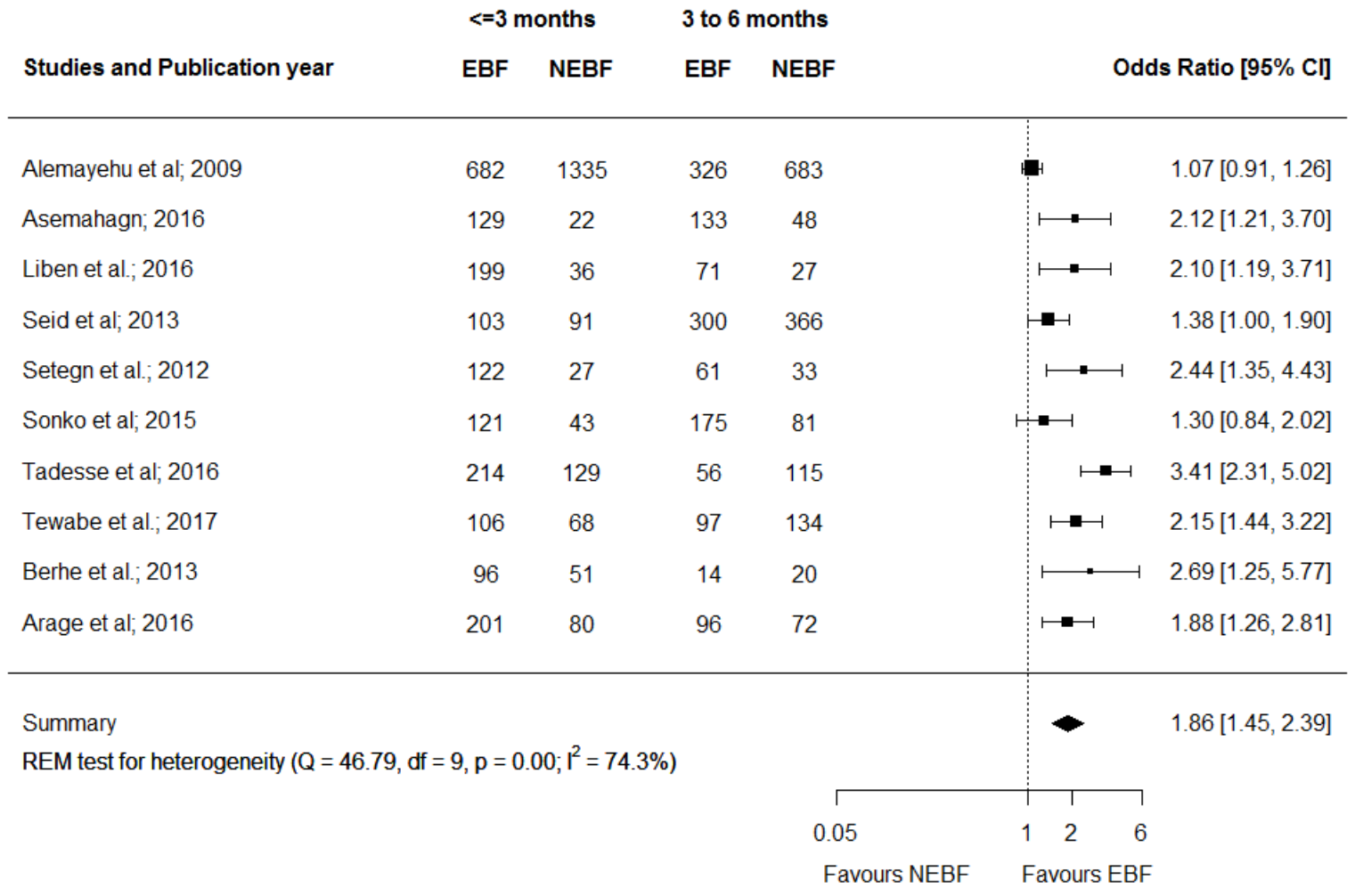

Figure 5: Forest plot of the unadjusted odds ratios with corresponding 95\% CIs of studies on the association of infant age and EBF. The horizontal line represents the confidence interval, the box and its size in the middle of the horizontal line represent the weight of sample size. The polygon represents the pooled odds ratio. $\mathrm{EBF}=$ Exclusive breastfeeding; $\mathrm{NEBF}=$ Non-exclusive of breastfeeding; REM=random effects model.

Finally, 12 studies ${ }^{(50,53,64,68,71-78)}$ reported the association between EBF and colostrum discarding with a sample of 6,035 mothers. As indicated in figure 6, the pooled OR of colostrum discarding was $0.56(95 \%$ CI $0.37-0.84, \mathrm{p}=0.005)$. Mothers who discard colostrum had $44 \%$ statistically significant lower chance of exclusively breastfeeding during the first 6 months compared to mothers who feed colostrum. Egger's regression test for funnel plot asymmetry was not significant $(\mathrm{z}=0.68, \mathrm{p}=0.49)$. 


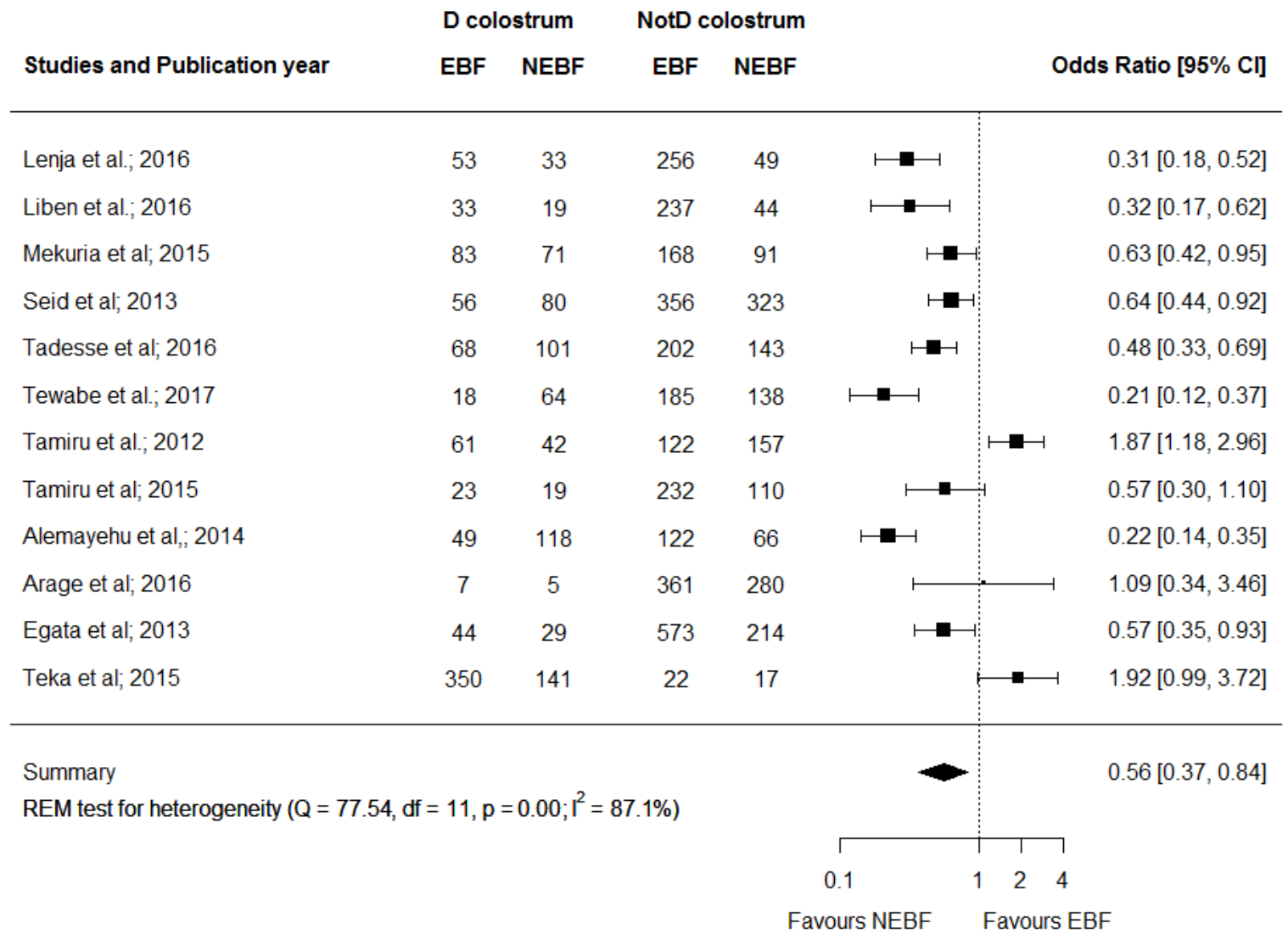

Figure 6: Forest plot of the unadjusted odds ratios with corresponding 95\% CIs of studies on the association of discarding colostrum and EBF. The horizontal line represents the confidence interval, the box and its size in the middle of the horizontal line represent the weight of sample size. The polygon represents the pooled odds ratio. $\mathrm{EBF}=$ Exclusive breastfeeding; $\mathrm{NEBF}=$ Non-exclusive breastfeeding; REM = random-effects model; D = Discarding; NotD = Not discarding. 


\section{Discussion}

This study examined the associations of TIBF and EBF with colostrum discarding, maternal/caregiver's age and infant age. To our knowledge, this is the first systematic review and meta-analyses in this topic to-date in Ethiopia. This meta-analysis uncovered colostrum discarding was significantly associated with TIBF but not maternal/caregiver's age. On the other hand, colostrum discarding and infant age was found to be significantly associated with EBF but not maternal/caregiver's age.

We found that colostrum discarding was significantly associated with TIBF. Mothers who discard colostrum had $62 \%$ significantly lower chance of initiating breastfeeding within one hour compared to mothers who feed colostrum to their child. This may be explained by the attempt of discard colostrum to get white milk may take time which therefore results in a delayed initiation of breastfeeding.

In the present meta-analysis, we found a statistically significant association between EBF and infant age. This finding confirmed our hypothesis and consistent with a large body of evidence showing that increased infant age is negatively associated with exclusive breastfeeding. ${ }^{(18,20,23,}$ 24,79,80) This may be due to the fact that giving traditional post-partum care and support is common in Ethiopia immediately after birth which may create opportunity for the mother to exclusively breastfeed the child. Since, this traditional post-partum care and support decreases as the age of the infant increases, it may lead the mother to work outside. This may therefore force the mother to stop EBF. Evidenced worldwide also agreed on the point that presence of social support is associated with better breastfeeding outcome. ${ }^{(81-83)}$ Another possible reason is the workload and short maternity leave in Ethiopia, which is only two months post-partum until recently, may influence the mother to withdraw EBF early. This hypothesis was supported by our previous meta-analyses whereby maternal employment significantly lower EBF and other studies. ${ }^{(83,84)}$ Moreover, this could also be related to the short birth interval in Ethiopia.

We noted that colostrum discarding significantly associated with EBF. The finding was in line with studies conducted in Nepal ${ }^{(85)}$ and Laos. ${ }^{(86)}$ This may be due to the fact that discarding colostrum leading to pre-lacteal feeding. In agreement with recent studies, ${ }^{(87-92)}$ maternal/caregiver's age was not significantly associated with either EBF or TIBF. This is against our hypothesis and disproves the notion that older mothers have better breastfeeding 
experience than young mothers that helps them to practice optimal TIBF and EBF. However, there is robust evidence that supported all reproductive age group mothers can maintain optimal TIBF and EBF equally. ${ }^{(24,84,93)}$ Therefore, the discrepancy may be due to the following reasons: (1) most studies used maternal age rather than age at first birth; (2) different studies have used different age categories; and (3) breastfeeding is not age dependent or can be confounded by innate maternal behavior.

This meta-analyses study has several implications. It provided evidence on breastfeeding practice and its associated factors in an Ethiopian context, which can be useful for cross-country / crosscultural comparison and for breastfeeding improvement initiative in Ethiopia. The present study provides an overview of up-to-date evidence for nutritionist and public health professionals. The findings also indicate emphasis should be given for all age group of mothers/caregivers during breastfeeding intervention. Furthermore, this study points out colostrum discarding and associated believes should be considered during designing breastfeeding interventions.

The association was estimated in large sample size and recent and nationally representative studies were included. In addition, this systematic review and meta-analysis was conducted based on a registered and published protocol, and guidelines for the Meta-analysis of Observational Studies in Epidemiology (MOOSE) was strictly followed. This study has also several limitations. First, some studies were excluded because of the difference in age category. Second, almost all included studies were observational which hinder causality inference. Third, even though we have used broad search strategies, the possibility of missing relevant studies cannot be fully exempted. Fourth, based on the conventional methods of statistical testing, a few analyses suffer from high levels of between-study heterogeneity. The course of heterogeneity was carefully explored and may be due to difference of study area; therefore, the result should be interpreted with caution.

In conclusion, colostrum discarding was a possible barrier for both TIBF and EBF. Additionally, increased infant age were found to be a risk factor for non EBF. However, maternal/caregiver's age was not a determinant factor for both TIBF and EBF. Interventions targeted on increasing the rate of TIBF and EBF should give special focus on colostrum discarding and associated beliefs. In addition, future research should be required to identify other factors affecting duration of EBF in Ethiopia. Further investigation is also required to assess the effect of age at first birth. 
Ethics approval: Not applicable.

Contributors: TD and SM conceived and designed the study. TD developed syntax for searching databases and analyzed the data. TD and SM wrote and revised the manuscript. All the authors read and have given the final approval.

Competing interests: None declared.

Funding: This study did not receive any specific grant from any funding agencies in the public, commercial, or not-for-profit sectors.

Patient Consent: Not required, because the review will not employ primary data collection. 


\section{Reference}

1. WHO. Indicators for assessing infant and young child feeding practices: part 1: definitions: conclusions of a consensus meeting held 6-8 November 2007 in Washington DC, USA. 2008.

2. WHO. Global strategy for infant and young child feeding: World Health Organization; 2003.

3. WHO. Complementary feeding: report of the global consultation, and summary of guiding principles for complementary feeding of the breastfed child. 2003.

4. WHO/UNICEF. GLOBAL BREASTFEEDING SCORECARD, 2017: Tracking Progress for Breastfeeding Policies and Programmes. 2017.

5. Chung M, Raman G, Chew P, Magula N, Trikalinos T, Lau J. Breastfeeding and maternal and infant health outcomes in developed countries. Evid Technol Asses (Full Rep). 2007;153(153):1-186.

6. Islam M, Rahman S, Kamruzzaman MI, Samad A. Effect of maternal status and breastfeeding practices on infant nutritional status-a cross sectional study in the south-west region of Bangladesh. The Pan African medical journal. 2013;16.

7. Patel DV, Bansal SC, Nimbalkar AS, Phatak AG, Nimbalkar SM, Desai RG. Breastfeeding practices, demographic variables, and their association with morbidities in children. Advances in preventive medicine. 2015;2015.

8. Edmond KM, Zandoh C, Quigley MA, Amenga-Etego S, Owusu-Agyei S, Kirkwood BR. Delayed breastfeeding initiation increases risk of neonatal mortality. Pediatrics. 2006;117(3):e380-e6.

9. UNICEF. The state of the world's children 2008: Child survival: Unicef; 2007.

10. Belfort MB. The Science of Breastfeeding and Brain Development. Breastfeeding Medicine. 2017;12(8):459-61.

11. UNICEF. Infant and young child feeding: Global Database. 2016.

12. Fisher J, Hammarberg K, Wynter K, McBain J, Gibson F, Boivin J, et al. Assisted conception, maternal age and breastfeeding: an Australian cohort study. Acta Paediatrica. 2013;102(10):970-6.

13. Amin T, Hablas H, Al Qader AA. Determinants of initiation and exclusivity of breastfeeding in Al Hassa, Saudi Arabia. Breastfeeding medicine. 2011;6(2):59-68. 
14. Esteves TMB, Daumas RP, Oliveira MICd, Andrade CAdFd, Leite IC. Factors associated to breastfeeding in the first hour of life: systematic review. Revista de saude publica. 2014;48(4):697-708.

15. Kaneko A, Kaneita Y, Yokoyama E, Miyake T, Harano S, Suzuki K, et al. Factors associated with exclusive breast-feeding in Japan: for activities to support child-rearing with breast-feeding. Journal of epidemiology. 2006;16(2):57-63.

16. Kitano N, Nomura K, Kido M, Murakami K, Ohkubo T, Ueno M, et al. Combined effects of maternal age and parity on successful initiation of exclusive breastfeeding. Preventive medicine reports. 2016;3:121-6.

17. Ludvigsson JF, Ludvigsson J. Socio $\square$ economic determinants, maternal smoking and coffee consumption, and exclusive breastfeeding in 10205 children. Acta paediatrica. 2005;94(9):1310-9.

18. Senarath U, Dibley MJ, Agho KE. Factors associated with nonexclusive breastfeeding in 5 east and southeast Asian countries: a multilevel analysis. Journal of Human Lactation. 2010;26(3):248-57.

19. Tarrant RC, Younger KM, Sheridan-Pereira M, White MJ, Kearney JM. The prevalence and determinants of breast-feeding initiation and duration in a sample of women in Ireland. Public health nutrition. 2010;13(6):760-70.

20. Victor R, Baines SK, Agho KE, Dibley MJ. Determinants of breastfeeding indicators among children less than 24 months of age in Tanzania: a secondary analysis of the 2010 Tanzania Demographic and Health Survey. BMJ open. 2013;3(1):e001529.

21. Meinzen-Derr JK, Guerrero ML, Altaye M, Ortega-Gallegos H, Ruiz-Palacios GM, Morrow AL. Risk of infant anemia is associated with exclusive breast-feeding and maternal anemia in a Mexican cohort. The Journal of nutrition. 2006;136(2):452-8.

22. Lande B, Andersen L, Baerug A, Trygg K, Lund $\square$ Larsen K, Veierød M, et al. Infant feeding practices and associated factors in the first six months of life: the Norwegian infant nutrition survey. Acta pædiatrica. 2003;92(2):152-61.

23. Nyanga NM, Musita C, Otieno A, Kaseje D. Factors influencing knowledge and practice of exclusive breastfeeding in Nyando district, Kenya. African journal of food, agriculture, nutrition and development. 2012;12(6). 
24. Patel A, Badhoniya N, Khadse S, Senarath U, Agho KE, Dibley MJ, et al. Infant and young child feeding indicators and determinants of poor feeding practices in India: secondary data analysis of National Family Health Survey 2005-06. Food and nutrition bulletin. 2010;31(2):314-33.

25. Legesse M, Demena M, Mesfin F, Haile D. Factors associated with colostrum avoidance among mothers of children aged less than 24 months in Raya Kobo district, North-eastern Ethiopia: community-based cross-sectional study. Journal of tropical pediatrics. 2015;61(5):35763.

26. Dennis CL. Breastfeeding initiation and duration: A $1990 \square 2000$ literature review. Journal of Obstetric, Gynecologic, \& Neonatal Nursing. 2002;31(1):12-32.

27. Hruschka DJ, Sellen DW, Stein AD, Martorell R. Delayed onset of lactation and risk of ending full breast-feeding early in rural Guatemala. The Journal of nutrition. 2003;133(8):25929.

28. Chye JK, Zain Z, Lim WL, Med M, Lim CT. Breastfeeding at 6 weeks and predictive factors. Journal of tropical pediatrics. 1997;43(5):287-92.

29. Koosha A, Hashemifesharaki R, Mousavinasab N. Breast-feeding patterns and factors determining exclusive breast-feeding. Singapore medical journal. 2008;49(12):1002.

30. Scott JA, Binns CW. Factors associated with the initiation and duration of breastfeeding: a review of the literature. Breastfeeding review: professional publication of the Nursing Mothers' Association of Australia. 1999;7(1):5-16.

31. Takahashi K, Ganchimeg T, Ota E, Vogel JP, Souza JP, Laopaiboon M, et al. Prevalence of early initiation of breastfeeding and determinants of delayed initiation of breastfeeding: secondary analysis of the WHO Global Survey. Scientific Reports. 2017;7.

32. Alebel A, Dejenu G, Mullu G, Abebe N, Gualu T, Eshetie S. Timely initiation of breastfeeding and its association with birth place in Ethiopia: a systematic review and metaanalysis. International breastfeeding journal. 2017;12(1):44.

33. Ethiopia M. National strategy for infant and young child feeding. 2004. p. 21.

34. Ethiopia FDR. National Guideline on Adolescent, Maternal, Infant and Young Child Nutrition. Addis Ababa, Ethiopia2016.

35. ETHIOPIA FDR. NATIONAL NUTRITION PROGRAM 2016-2020. Addis Ababa, Ethiopia2016. 
36. Ethiopia FDR. National Nutrition Programme June 2013 - June 2015. Addis Ababa, Ethiopia2013.

37. MoH EFDR. Health Sector Transformation Plan 2015/16 - 2019/2020(2008-2012 EFY). 2015.

38. UNICEF. News Note: World Breastfeeding Week Celebrated in Ethiopia 2009.

39. Habtewold TD, Islam MA, Sharew NT, Mohammed SH, Birhanu MM, Tegegne BS.

SystEmatic review and meta-aNAlysis of infanT and young child feeding Practices (ENAT-P) in Ethiopia: protocol. BMJ Open. 2017;7(8).

40. Hartling L, Hamm M, Milne A, Vandermeer B, Santaguida PL, Ansari M, et al. Validity and inter-rater reliability testing of quality assessment instruments. 2012.

41. Hootman JM, Driban JB, Sitler MR, Harris KP, Cattano NM. Reliability and validity of three quality rating instruments for systematic reviews of observational studies. Research synthesis methods. 2011;2(2):110-8.

42. Boccolini CS, Carvalho ML, Oliveira MI. Factors associated with exclusive breastfeeding in the first six months of life in Brazil: a systematic review. Rev Saude Publica. $2015 ; 49$.

43. Sharma IK, Byrne A. Early initiation of breastfeeding: a systematic literature review of factors and barriers in South Asia. International breastfeeding journal. 2016;11(1):17.

44. Egger M, Smith GD, Schneider M, Minder C. Bias in meta-analysis detected by a simple, graphical test. Bmj. 1997;315(7109):629-34.

45. Duval S, Tweedie R. Trim and fill: a simple funnel $\square$ plot-based method of testing and adjusting for publication bias in meta $\square$ analysis. Biometrics. 2000;56(2):455-63.

46. Wolde T, Birhanu T, Ejeta E. Prevalence and determinants of timely initiation of breastfeeding among lactating mothers of urban dwellers in Western Ethiopia: A community based cross sectional study. Food Science and Quality Management. 2014;31.

47. Woldemichael B, Kibie Y. Timely Initiation of Breastfeeding and Its Associated Factors among Mothers in Tiyo Woreda, Arsi Zone, Ethiopia: A Community-Based Cross Sectional Study. Clinics Mother Child Health. 2016;13(221):2.

48. Adugna DT. Women's perception and risk factors for delayed initiation of breastfeeding in Arba Minch Zuria, Southern Ethiopia. International breastfeeding journal. 2014;9(1):8. 
49. Beyene MG, Geda NR, Habtewold TD, Assen ZM. Early initiation of breastfeeding among mothers of children under the age of 24 months in Southern Ethiopia. International breastfeeding journal. 2017;12(1):1.

50. Alemayehu M, Abreha K, Yebyo H, Zemichael K, Gebremichael H. Factors associated with timely initiation and exclusive breast feeding among mothers of Axum town, Northern Ethiopia. Sci J Public Health. 2014;2(5):394-401.

51. Berhe H, Mekonnen B, Bayray A, Berhe H. Determinants of Breast feeding Practices Among Mothers Attending Public Health Facilities, Mekelle, Northern Ethiopia; A Cross Sectional Study. International Journal of Pharmaceutical Sciences and Research. 2013;4(2):650. 52. Setegn T, Gerbaba M, Belachew T. Determinants of timely initiation of breastfeeding among mothers in Goba Woreda, South East Ethiopia: A cross sectional study. BMC public health. 2011;11(1):217.

53. Tamiru D, Tamrat M. Constraints to the optimal breastfeeding practices of breastfeeding mothers in the rural communities of Arba Minch Zuria Woreda, Ethiopia: a community-based, cross-sectional study. South African Journal of Clinical Nutrition. 2015;28(3):134-9.

54. Regassa N. Infant and child feeding practices among farming communities in Southern Ethiopia. Kontakt. 2014;16(4):e215-e22.

55. Ekubay M, Berhe A, Yisma E. Initiation of breastfeeding within one hour of birth among mothers with infants younger than or equal to 6 months of age attending public health institutions in Addis Ababa, Ethiopia. International Breastfeeding Journal. 2018;13(1):4.

56. Hailemariam TW, Adeba E, Sufa A. Predictors of early breastfeeding initiation among mothers of children under 24 months of age in rural part of West Ethiopia. BMC public health. 2015;15(1):1076.

57. Tewabe T. Timely initiation of breastfeeding and associated factors among mothers in Motta town, East Gojjam zone, Amhara regional state, Ethiopia, 2015: a cross-sectional study. BMC pregnancy and childbirth. 2016;16(1):314.

58. Tilahun G, Degu G, Azale T, Tigabu A. Prevalence and associated factors of timely initiation of breastfeeding among mothers at Debre Berhan town, Ethiopia: a cross-sectional study. International breastfeeding journal. 2016;11(1):27. 
59. Liben ML, Yesuf EM. Determinants of early initiation of breastfeeding in Amibara district, Northeastern Ethiopia: a community based cross-sectional study. International breastfeeding journal. 2016;11(1):7.

60. Abera K. Infant and young child feeding practices among mothers living in Harar, Ethiopia. Harar Bulletin of Health Sciences. 2012;4:66-78.

61. Getahun EA, Hayelom DH, Kassie GG. Exclusive Breast Feeding Practice and Associated Factors in Kemba Woreda, Southern Ethiopia, a Community Based Cross-Sectional Study. International Journal of Science, Technology and Society. 2017;5(4):55.

62. Gizaw Z, Woldu W, Bitew BD. Exclusive breastfeeding status of children aged between 6 and 24 months in the nomadic population of Hadaleala district, Afar Region, northeast Ethiopia. International breastfeeding journal. 2017;12(1):38.

63. Hunegnaw MT, Gezie LD, Teferra AS. Exclusive breastfeeding and associated factors among mothers in Gozamin district, northwest Ethiopia: a community based cross-sectional study. International breastfeeding journal. 2017;12(1):30.

64. Lenja A, Demissie T, Yohannes B, Yohannis M. Determinants of exclusive breastfeeding practice to infants aged less than six months in Offa district, Southern Ethiopia: a cross-sectional study. International breastfeeding journal. 2016;11(1):32.

65. Setegn T, Belachew T, Gerbaba M, Deribe K, Deribew A, Biadgilign S. Factors associated with exclusive breastfeeding practices among mothers in Goba district, south east Ethiopia: a cross-sectional study. International breastfeeding journal. 2012;7(1):17.

66. Sonko A, Worku A. Prevalence and predictors of exclusive breastfeeding for the first six months of life among women in Halaba special woreda, Southern Nations, Nationalities and Peoples' Region/SNNPR/, Ethiopia: a community based cross-sectional study. Archives of Public Health. 2015;73(1):53.

67. Asfaw MM, Argaw MD, Kefene ZK. Factors associated with exclusive breastfeeding practices in Debre Berhan District, Central Ethiopia: a cross sectional community based study. International breastfeeding journal. 2015;10(1):23.

68. Teka B, Assefa H, Haileslassie K. Prevalence and determinant factors of exclusive breastfeeding practices among mothers in Enderta woreda, Tigray, North Ethiopia: a crosssectional study. International breastfeeding journal. 2015;10(1):2. 
69. Alemayehu T, Haidar J, Habte D. Determinants of exclusive breastfeeding practices in Ethiopia. Ethiopian Journal of Health Development. 2009;23(1).

70. Asemahagn MA. Determinants of exclusive breastfeeding practices among mothers in azezo district, northwest Ethiopia. International breastfeeding journal. 2016;11(1):22.

71. Liben ML, Gemechu YB, Adugnew M, Asrade A, Adamie B, Gebremedin E, et al. Factors associated with exclusive breastfeeding practices among mothers in dubti town, afar regional state, northeast Ethiopia: a community based cross-sectional study. International breastfeeding journal. 2016;11(1):4.

72. Seid AM, Yesuf ME, Koye DN. Prevalence of Exclusive Breastfeeding Practices and associated factors among mothers in Bahir Dar city, Northwest Ethiopia: a community based cross-sectional study. International breastfeeding journal. 2013;8(1):14.

73. Tadesse T, Mesfin F, Chane T. Prevalence and associated factors of non-exclusive breastfeeding of infants during the first six months in rural area of Sorro District, Southern Ethiopia: a cross-sectional study. International breastfeeding journal. 2016;11(1):25.

74. Tewabe T, Mandesh A, Gualu T, Alem G, Mekuria G, Zeleke H. Exclusive breastfeeding practice and associated factors among mothers in Motta town, East Gojjam zone, Amhara Regional State, Ethiopia, 2015: a cross-sectional study. International breastfeeding journal. 2017;12(1):12.

75. Arage G, Gedamu H. Exclusive Breastfeeding Practice and Its Associated Factors among Mothers of Infants Less Than Six Months of Age in Debre Tabor Town, Northwest Ethiopia: A Cross-Sectional Study. Advances in Public Health. 2016;2016.

76. Egata G, Berhane Y, Worku A. Predictors of non-exclusive breastfeeding at 6 months among rural mothers in east Ethiopia: a community-based analytical cross-sectional study. International breastfeeding journal. 2013;8(1):8.

77. Mekuria G, Edris M. Exclusive breastfeeding and associated factors among mothers in Debre Markos, Northwest Ethiopia: a cross-sectional study. International breastfeeding journal. 2015;10(1):1.

78. Tamiru D, Belachew T, Loha E, Mohammed S. Sub-optimal breastfeeding of infants during the first six months and associated factors in rural communities of Jimma Arjo Woreda, Southwest Ethiopia. BMC Public Health. 2012;12(1):363. 
79. Dorgham L, Hafez S, Kamhawy H, Hassan W. Assessment of initiation of breastfeeding, prevalence of exclusive breast feeding and their predictors in Taif, KSA. Life Sci J. 2014;11:1.

80. Agho KE, Dibley MJ, Odiase JI, Ogbonmwan SM. Determinants of exclusive breastfeeding in Nigeria. BMC pregnancy and childbirth. 2011;11(1):2.

81. Kanhadilok S, McGrath JM. An integrative review of factors influencing breastfeeding in adolescent mothers. The Journal of perinatal education. 2015;24(2):119.

82. Emmanuel A. A literature review of the factors that influence breastfeeding: An application of the health belief model. 2015.

83. Balogun OO, Dagvadorj A, Anigo KM, Ota E, Sasaki S. Factors influencing breastfeeding exclusivity during the first 6 months of life in developing countries: a quantitative and qualitative systematic review. Maternal \& child nutrition. 2015;11(4):433-51.

84. Pereira-Santos M, Santana MdS, Oliveira DS, Nepomuceno Filho RA, Lisboa CS, Almeida LMR, et al. Prevalence and associated factors for early interruption of exclusive breastfeeding: meta-analysis on Brazilian epidemiological studies. Revista Brasileira de Saúde Materno Infantil. 2017;17(1):59-67.

85. Chandrashekhar T, Joshi H, Binu V, Shankar P, Rana M, Ramachandran U. Breastfeeding initiation and determinants of exclusive breast-feeding-a questionnaire survey in an urban population of western Nepal. Public health nutrition. 2007;10(2):192-7.

86. Barennes H, Empis G, Quang TD, Sengkhamyong K, Phasavath P, Harimanana A, et al. Breast-milk substitutes: a new old-threat for breastfeeding policy in developing countries. A case study in a traditionally high breastfeeding country. PloS one. 2012;7(2):e30634.

87. Radwan H. Patterns and determinants of breastfeeding and complementary feeding practices of Emirati Mothers in the United Arab Emirates. BMC public health. 2013;13(1):171.

88. Ghwass MMA, Ahmed D. Prevalence and predictors of 6-month exclusive breastfeeding in a rural area in Egypt. Breastfeeding medicine. 2011;6(4):191-6.

89. Yılmaz E, Öcal FD, Yılmaz ZV, Ceyhan M, Kara OF, Küçüközkan T. Early initiation and exclusive breastfeeding: Factors influencing the attitudes of mothers who gave birth in a baby-friendly hospital. Turkish journal of obstetrics and gynecology. 2017;14(1):1.

90. El-Gilany AH, El-Wehady A, El-Hawary A. Maternal employment and maternity care in Al-Hassa, Saudi Arabia. The European Journal of Contraception \& Reproductive Health Care. 2008;13(3):304-12. 
91. Ogunlesi TA. Maternal socio-demographic factors influencing the initiation and exclusivity of breastfeeding in a Nigerian semi-urban setting. Maternal and child health journal. 2010;14(3):459-65.

92. Pandey S, Tiwari K, Senarath U, Agho KE, Dibley MJ, * SAIFRN. Determinants of infant and young child feeding practices in Nepal: secondary data analysis of Demographic and Health Survey 2006. Food and nutrition bulletin. 2010;31(2):334-51.

93. Perera PJ, Ranathunga N, Fernando MP, Sampath W, Samaranayake GB. Actual exclusive breastfeeding rates and determinants among a cohort of children living in Gampaha district Sri Lanka: A prospective observational study. International breastfeeding journal. 2012;7(1):21. 